\title{
Fiabilidad y precisión en Biomecánica
}

\section{Sáenz-Nuño, M. A. ${ }^{a^{*}}$; Pérez-Mallada, N.b; Díez-Sánchez, N.c; Rodeño-Domínguez, M.} ; Moreno-Bellido, F $^{\text {c }}$

${ }^{a}$ Dpto. Ing. Mecánica, Escuela Técnica Superior de Ingeniería, ICAI, Univ. Pontificia Comillas de Madrid,msaenz@comillas.edu,

${ }^{\mathrm{b}}$ Escuela de Enfermería y Fisioterapia San Juan de Dios, Univ. Pontificia Comillas de Madrid, ${ }^{\mathrm{c}}$ Laboratorio de Metrología Dimensional de FREMAP. Servicio de Readaptación Profesional, Hospital de Majadahonda.

\begin{abstract}
There has been developed a lot of new systems for the diagnosis of different illnesses or the measurement of physical parameters. All of them integrated in those small wearables. These equipments allow to have a complete info of the biomechanical variables of interest, without interfering in the diagnosis.

In order to develop realistic and objective models for biomechanical it is is been developed reliable measurements with traceability to the main standards.

In this paper it is presented the successful calibration procedure for a digital goniometer, it has been metrologically characterized, assessing its measurements and estableshing it precision range and accuracy.

This research is the result of a collaboration between an ENAC accredited laboratory and the Universidad Pontificia Comillas, where the system was developed together with other partner.
\end{abstract}

Keywords: Fiability, precision, accuracy, comparability.

\section{Resumen}

Son evidentes las mejoras que se están realizando en los sistemas de diagnóstico utilizados en Biomecánica, con equipos cada vez más integrados en la actividad física a través de diminutos wearables. Ello permite hacer seguimiento de las variables biomecánicas de interés, sin apenas interferir y por consiguiente mejorar la objetividad de los diagnósticos.

Si a esto se aúna la seguridad de unas medidas fiables trazadas a referencias internacionales, se podrán obtener modelos objetivos y realistas biomecánicos totalmente a medida. En el presente trabajo se presenta la experiencia de calibración de un goniómetro digital para su caracterización metrológica, asegurando sus resultados y estableciendo su rango de 
precisión y exactitud. Dicho trabajo se realizó en un laboratorio acreditado por ENAC y con una clara implicación en el sector sanitario. Con ello, todas las mediciones realizadas con este goniómetro van a ofrecer una fiabilidad certificada dentro incluso del sistema industrial, que permitirá garantizar un seguimiento, objetivo y comparable, en la evolución del tratamiento, valoración funcional y pericial de un paciente.

Se presentará también la propuesta de Gestión Metrológica para el equipamiento de medición en Biomecánica, que actualmente se está liderando desde UNE en conjunción con el CEM, Comillas, FREMAP y otras instituciones

Palabras clave: Fiabilidad, precisión, exactitud, comparabilidad

\section{Introducción}

Si bien la medición de ángulos es utilizada desde hace mucho tiempo en los protocolos de análisis de la marcha entre otros, no es muy habitual disponer de trazabilidad en las mediciones. Con el uso de acelerómetros integrados en dispositivos wearables, su uso se ha extendido en la práctica.

En este artículo presentamos la calibración realizada por un laboratorio acreditado en la red ENAC para la completa caracterización del equipo, poniendo de manifiesto la sencillez en la integración del sistema en la cadena de trazabilidad, asegurando así la fiabilidad de sus mediciones.

\section{Goniómetro HAWK para la medición en fisioterapia y el deporte.}

El goniómetro HAWK es un equipo especialmente diseñado para la medición de ángulos en el movimiento del cuerpo humano con una resolución de $1^{\circ}$, siendo un sustituto ideal de los goniómetros tradicionales de división de escala de $5^{\circ}$. Este equipo también se puede utilizar industrialmente para la medición de cualquier ángulo.

Con este estudio se ha caracterizado el funcionamiento del equipo y su respuesta tanto en medición sobre diferentes medios, como el resultado de una medida cuando han transcurrido varios días. De esta forma, se proporciona un ejemplo a seguir para el aseguramiento de las mediciones en otros equipos de uso similar.

\subsection{Descripcion del equipo y su funcionamiento.}

El sensor es un cuerpo de plástico en forma de paralelepípedo que capta el movimiento angular cuando se desplaza en cualquiera de los tres ejes coordenados X, Y y Z (figura 1). 
Si se desplaza el cuerpo de medida en un movimiento angular, éste capta el ángulo mediante un giróscopo interno (según la información facilitada por el fabricante). Esta medición angular se envía por bluetooth a un teléfono móvil que tiene cargada la aplicación del fabricante (APP) para este dispositivo y nos muestra en pantalla los tres ángulos del movimiento realizado, ángulo en relación al eje $\mathrm{X}$, eje $\mathrm{Y}$ y eje $\mathrm{Z}$.
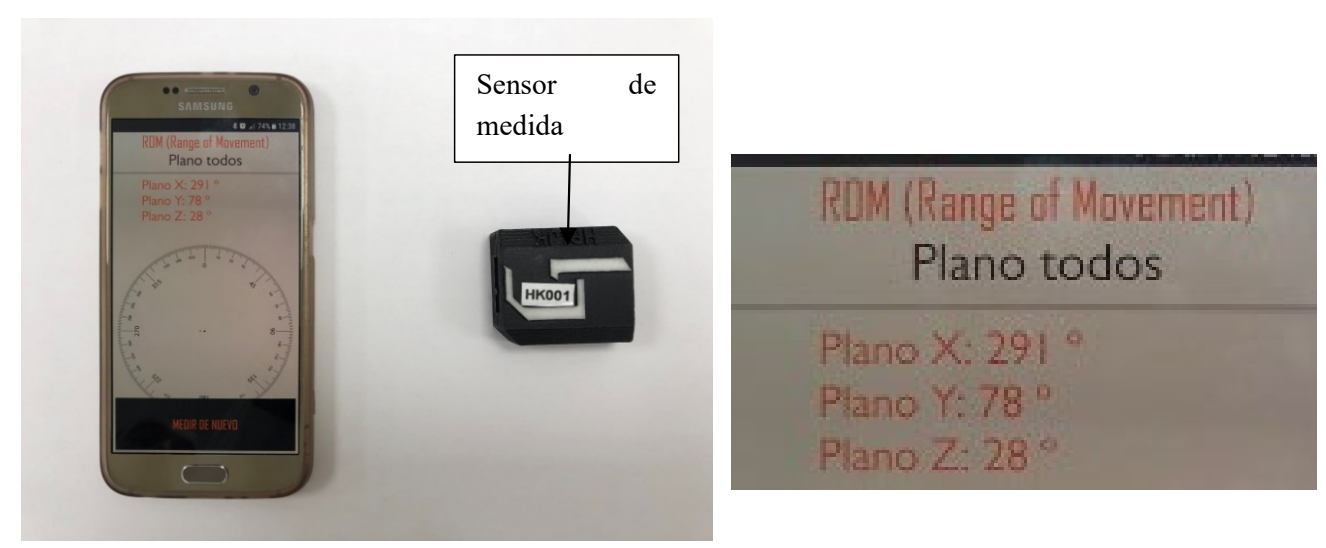

Fig. 1 HAWK. Fuente: Imagen propia (2019).

El equipo tiene un rango de medida de $360^{\circ}$, una división de escala de $1^{\circ}$ y unas dimensiones externas de $43 \times 38 \times 17 \mathrm{~mm}$.

\section{Referencias angulares para la calibración.}

Para realizar el estudio del goniómetro nos planteamos diferentes escenarios de trabajo, de tal manera que pudiéramos ver los errores e incertidumbre de cada uno de ellos y poder concluir con la incertidumbre del equipo en el caso más eficaz, para definir el procedimiento de calibración a realizar. Se hicieron cuatro pruebas:

\subsection{Medida de ángulos sobre plataforma móvil situada en máquina de medida por 3 coordenadas}

Con este ensayo se reprodujo lo más fehacientemente el movimiento del goniómetro sobre una superficie similar a un brazo humano, que es la medida de la plataforma de giro.

La máquina de tres coordenadas trabaja como referencia para la calibración del goniómetro. 

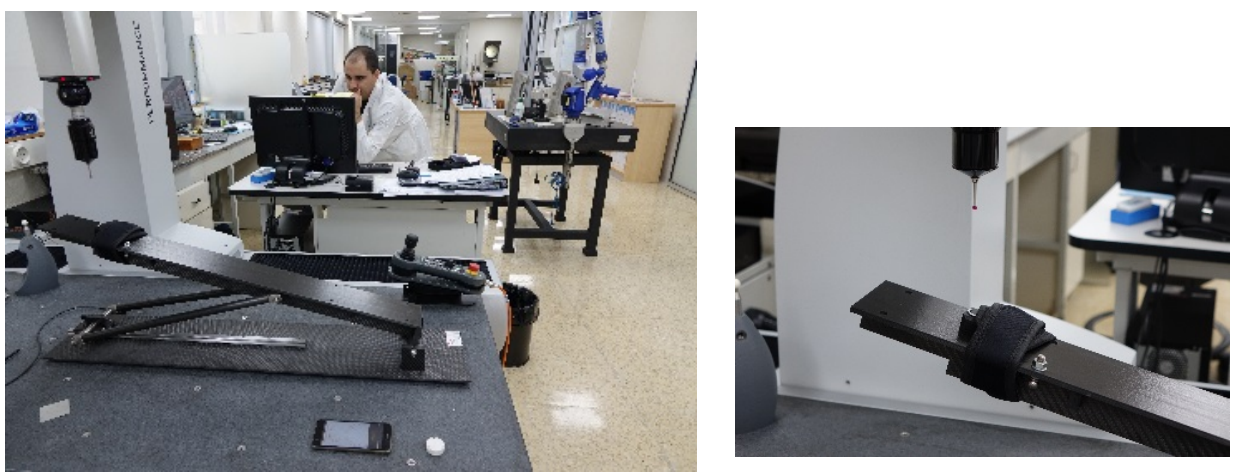

Fig. 2. Medición en la máquina de medición por coordenadas en el laboratorio acreditado de FREMAP. Fuente: Imagen propia (2018).

- Se sujetó el goniómetro sobre la plataforma móvil -- base con giro utilizada para el patrón de bolas-.

- Se midió con la máquina de tres coordenadas calibrada un plano de referencia $0^{\circ}$ sobre la cara del cuerpo del goniómetro, una vez fijado sobre la plataforma móvil, tal y como se aprecia en la figura 2.

- Se colocó la plataforma en posición $10^{\circ}$, según el indicador del mensurando, y se realizó la medición de un plano en la misma zona que el de referencia (se fue abriendo el ángulo de la plataforma hasta que la APP del HAWK indicaba $10^{\circ}$, se fijaba la plataforma y se medía el ángulo con la máquina 3D).

- Se repitió el proceso anterior con los ángulos de $20^{\circ}$ y $30^{\circ}$.

*Nota: las mediciones en la medidora de tres coordenadas se realizaron en modo automático.

\subsection{Medida de ángulos en un solo eje situado sobre proyector de perfiles.}

En este caso, se procedió a la calibración individual de cada eje del HAWK frente a un proyector de perfiles, otro equipo también calibrado y con amplia y variada aplicación en la industria. Con este ensayo se estudió cada uno de los ejes de medición del goniómetro, sin interferencia de medida de los otros ángulos.

Al posicionar el cuerpo del goniómetro sobre la pantalla giratoria del proyector de perfiles, el HAWK medía ángulos en uno solo de los tres ejes X, Y, Z, dependiendo de la superficie del mismo que se apoyase en la pantalla del proyector de perfiles.

Para ello, una vez posicionado el cuerpo del goniómetro sobre la pantalla giratoria del proyector de perfiles, se giraba la pantalla hasta conseguir el ángulo deseado en el HAWK ( $\mathrm{p}$ ej. $35^{\circ}$ ) y leyendo el ángulo en la APP del móvil enlazado al HAWK y que actuaba como 
display del mismo. El ángulo patrón era el del giro de la pantalla del proyector de perfiles y con resolución de 1 minuto.

Las pautas que se han seguido para realizar este estudio fueron las siguientes:

- Se eligía la cara principal del HAWK y se colocaba sobre la pantalla giratoria del proyector de perfiles.

- Se escogieron al azar 5 ángulos puros, para ello se giró la pantalla del proyector de perfiles hasta que la APP del HAWK marcaba el ángulo a medir: $10^{\circ}, 45^{\circ}, 65^{\circ}, 72^{\circ} \mathrm{y}$ $88^{\circ}$, manteniendo la pantalla inmóvil se tomó un ángulo de giro que fuera el ángulo patrón. Se repitió la operación cuatro veces por ángulo y el juego de medida se repitió tres veces en el transcurso de una hora.
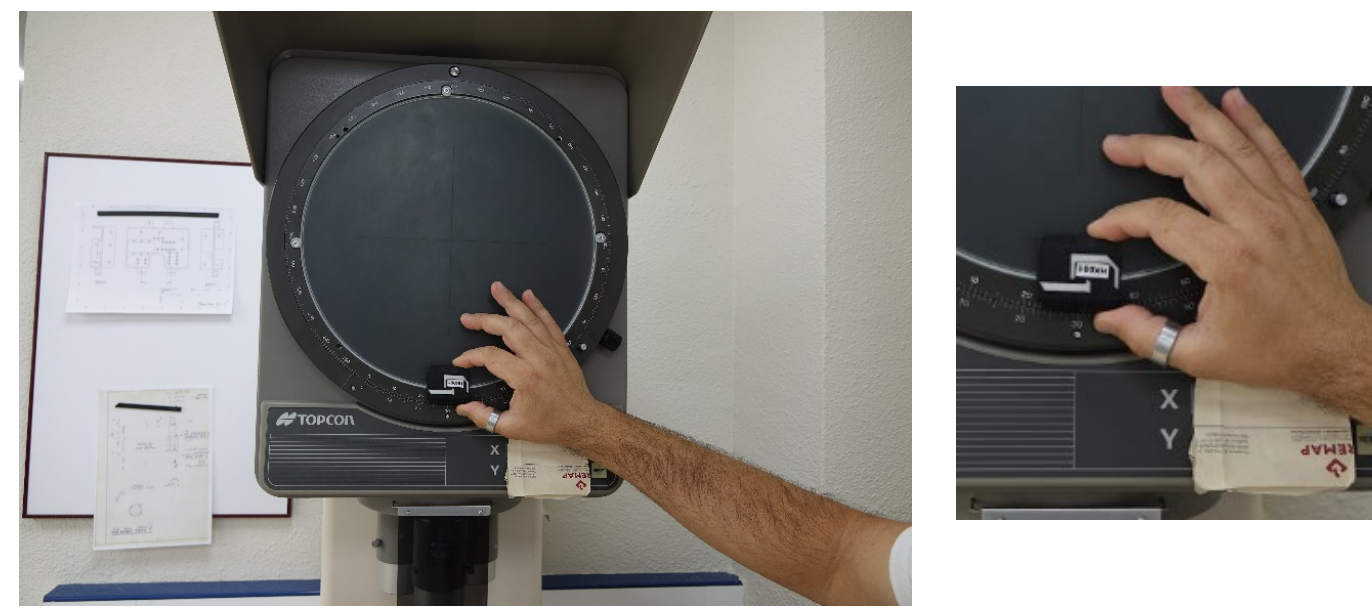

Fig. 3. Medición en el proyector de perfiles en el laboratorio acreditado de FREMAP.

Fuente: Imagen propia (2018).

\subsection{Reproducibilidad del HAWK con proyector de perfiles.}

Se realizó en días diferentes para comprobar si el tiempo entre medida influía en el resultado, asegurando así un valor máximo para la reproducibilidad del equipo.

Siguiendo el esquema de medición de 2.2 se tomaron medidas sobre los ángulos de $72^{\circ} \mathrm{y}$ $185^{\circ}$ en tres días consecutivos.

Se compararon los resultados: medias aritméticas y desviaciones típicas de las 10 medidas repetidas para cada ángulo. 


\subsection{Medida de ángulos sobre proyector de perfiles, posicionando el HAWK en cada una de sus 6 caras y en giro de $90^{\circ}$ en cada una de las caras.}

Se realizó este estudio, con el fin de conocer si la posición del cuerpo del goniómetro afectaba a los resultados de la medición, comprobándose que no era relevante.

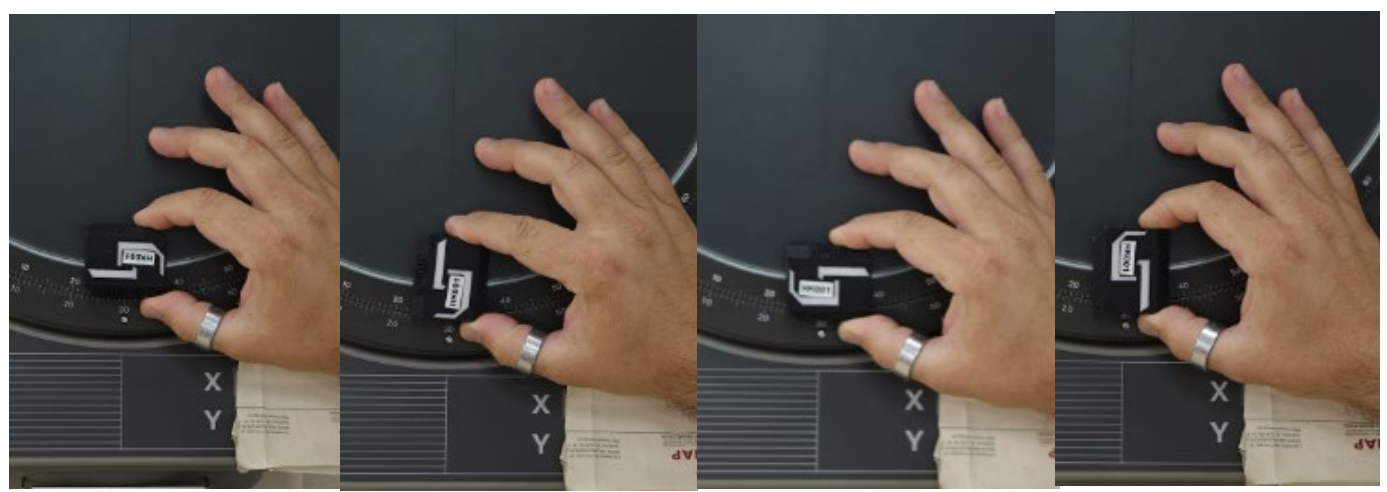

Fig. 4. Medición en el proyector de perfiles en el laboratorio acreditado de FREMAP sobre todas las caras del HAWK (sólo se muestran algunas posiciones).Fuente: Imagen propia (2018).

\section{Adquisición de datos en todos los casos.}

En cada uno de los procedimientos anteriores, la obtención de las lecturas con el equipo fueron:

- Se instaló en un Smartphone el software GONIOMETRO ADVANCE Versión 7.0, disponible en Play Store de Android y que actúa como display del equipo.

- El equipo estaba con la batería cargada al 100\% al inicio de cada serie de medidas.

- Se encendía el goniómetro y se enlazaba mediante bluetooth con la aplicación del Smartphone.

- Una vez encendido y enlazado el equipo, se esperaba cinco minutos para que este se atemperase.

- Se verificaba que el equipo medía en todo su rango, esto es, de $0^{\circ}$ a $360^{\circ}$.

\section{Resultados preliminares.}

Los resultados obtenidos con la máquina de medición por coordenadas en tres ángulos, dieron como resultado una corrección máxima de $0,5^{\circ} \mathrm{y}$ una desviación típica inferior a $0,3^{\circ}$ (véase la tabla 1).

Tabla 1. Medida en grados en la máquina de 3 coordenadas 
Sáenz-Nuño, M. A.; Pérez-Mallada, N.; Díez-Sánchez, N.; Rodeño-Domínguez,M; Moreno-Bellido, F.

\begin{tabular}{|c|c|c|c|c|c|c|c|c|c|}
\hline COTAS & NOMINAL & $\begin{array}{c}\text { MEDIDA } \\
1 \\
\end{array}$ & $\begin{array}{c}\text { MEDIDA } \\
2 \\
\end{array}$ & $\begin{array}{c}\text { MEDIDA } \\
\mathbf{3} \\
\end{array}$ & $\begin{array}{c}\text { MEDIDA } \\
4 \\
\end{array}$ & $\begin{array}{c}\text { MEDIDA } \\
5 \\
\end{array}$ & MEDIA $\square$ & $\begin{array}{c}\text { DES. } \\
\text { TIP }\end{array}$ & $\begin{array}{c}\square \\
\text { CORREC }\end{array}$ \\
\hline 1 & 10 & 9,6108 & 10,1411 & 9,8872 & 9,8691 & 9,5561 & 9,81286 & 0,236212 & $-0,18706$ \\
\hline 2 & 20 & 19,8205 & 19,9588 & 19,848 & 19,9413 & 19,5166 & 19,81704 & 0,178025 & $-0,18286$ \\
\hline 3 & 30 & 29,5255 & 29,5563 & 29,7972 & 29,2877 & 29,3525 & 29,50384 & 0,199312 & $-0,49608$ \\
\hline & & & \multicolumn{3}{|c|}{ MAX DESVIACION TIPICA } & 0,236212 & & & \\
\hline & & & \multicolumn{3}{|c|}{ MAX CORRECCION } & $-0,4960$ & & & \\
\hline
\end{tabular}

Los resultados obtenidos con el proyector de perfiles, dieron como resultado una corrección inferior a $1,5^{\circ}$ y una desviación típica inferior a $1^{\circ}$ (véanse las tablas 2 ).

Tabla 2. Medida de ángulos en un solo eje situado sobre proyector de perfiles en cada eje

\begin{tabular}{|c|c|c|c|c|c|}
\hline \multirow{2}{*}{$\begin{array}{l}\text { Medidas } \\
\text { Eje X }\end{array}$} & \multicolumn{5}{|c|}{ ANGULOS PUROS EN GRADOS : ${ }^{\circ}$} \\
\hline & $10^{\circ}$ & 45 & 65 & 72 & 88 \\
\hline 1 & 10 & 44,766 & 63,816 & 70,416 & 86,783 \\
\hline 2 & 10 & 44,200 & 63,450 & 71,233 & 86,400 \\
\hline 3 & 10 & 44,600 & 63,566 & 70,350 & 86,916 \\
\hline 4 & 10,160 & 43,833 & 63,816 & 70,400 & 86,450 \\
\hline media & 10,040 & 44,350 & 63,662 & 70,600 & 86,637 \\
\hline D. Típica/ & 0,0800 & 0,4185 & 0,1840 & 0,4231 & 0,2519 \\
\hline d. típica/' & 4,800 & 25,108 & 11,041 & 25,386 & 15,111 \\
\hline corrección & $-0,040$ & 0,650 & 1,338 & 1,400 & 1,363 \\
\hline
\end{tabular}

\begin{tabular}{|c|c|c|c|c|c|}
\hline \multirow{2}{*}{$\begin{array}{l}\text { Medidas } \\
\text { Eje Y }\end{array}$} & \multicolumn{5}{|c|}{ ANGULOS PUROS EN GRADOS : ${ }^{\circ}$} \\
\hline & 10 & 45 & 65 & 72 & 88 \\
\hline 1 & 9,933 & 44,416 & 63,500 & 70,666 & 86,966 \\
\hline 2 & 10,200 & 44,583 & 63,616 & 70,616 & 87,583 \\
\hline 3 & 9,900 & 44,566 & 64,250 & 70,250 & 86,450 \\
\hline 4 & 9,916 & 44,416 & 63,583 & 70,583 & 86,333 \\
\hline media & 9,987 & 44,495 & 63,737 & 70,529 & 86,833 \\
\hline D. Típica ${ }^{\circ}$ & 0,1425 & 0,0918 & 0,3453 & 0,1889 & 0,5706 \\
\hline d. típica/' & 8,548 & 5,506 & 20,718 & 11,336 & 34,238 \\
\hline Corrección & 0,013 & 0,505 & 1,263 & 1,471 & 1,167 \\
\hline
\end{tabular}




\begin{tabular}{|c|c|c|c|c|c|}
\hline \multirow{2}{*}{$\begin{array}{l}\text { Medidas } \\
\text { Eje Z }\end{array}$} & \multicolumn{5}{|c|}{ ANGULOS PUROS EN GRADOS : ${ }^{\circ}$} \\
\hline & 10 & 45 & 65 & 72 & 88 \\
\hline 1 & 9,900 & 45,116 & 64,516 & 72,000 & 87,316 \\
\hline 2 & 10,083 & 44,900 & 64,666 & 71,566 & 86,850 \\
\hline 3 & 10,083 & 44,716 & 64,683 & 71,683 & 87,616 \\
\hline 4 & 10,083 & 44,650 & 64,500 & 71,866 & 87,150 \\
\hline Media & 10,037 & 44,846 & 64,591 & 71,779 & 87,233 \\
\hline D. Típica/ ${ }^{\circ}$ & 0,0915 & 0,2091 & 0,0966 & 0,1923 & 0,3200 \\
\hline d. típica/ ' & 5,490 & 12,544 & 5,796 & 11,541 & 19,199 \\
\hline corrección & $-0,037$ & 0,154 & 0,409 & 0,221 & 0,767 \\
\hline
\end{tabular}

Una vez realizado el estudio con el proyector de perfiles, observando los resultados y comparándolos con los del estudio de la máquina de coordenadas, llegamos a la conclusión de seguir realizando el estudio con el proyector de perfiles como patrón ya que con este la incertidumbre que se obtiene es del orden de magnitud de la división de escala, y por tanto, suficiente para las prestaciones del equipo. Suponiendo además un coste significativo en la calibración

Después de haber analizado los resultados obtenidos sobre las medidas realizadas en tres días diferentes consecutivos, llegamos a la conclusión de que el paso del tiempo no influye en el equipo y que por lo tanto esto no influirá en su incertidumbre final. Por lo tanto se consideró que la contribución por reprodubilidad era nula.

\section{Resultados finales.}

La calibración del HAWK condujo así a los siguientes resultados:

Tabla 3. Resultados de la calibración

\begin{tabular}{cc}
\hline Eje & Incertidumbre (k=2) \\
\hline Eje X & $2^{\circ} 18^{\prime}$ \\
Eje Y & $1^{\circ} 4^{\prime}$ \\
Eje Z & $1^{\circ} 1^{\prime}$ \\
\hline
\end{tabular}




\section{Conclusiones.}

Se demuestra la viabilidad de la calibración de instrumentos denominados wearables, con trazabilidad a la cadena industrial, con procedimientos sencillos y fiables.

Se recomienda la utilización de este tipo de procesos para asegurar unas mediciones en el ámbito de la salud, proponiendo la elaboración de nuevos procesos para el entorno sanitario, como es el caso de la Gestión Metrológica Sanitaria.

\section{Referencias}

A. Marín Fernández-Sánchez, A. López-Díaz, C. Jiménez- Alonso, M. Sáenz, J. de Vicente y Oliva. Análisis de las carencias en normalización para la trazabilidad metrológica de equipos médicos. Dyna. vol. 91, no. 5, pp. 536-540, Septiembre 2016. JCR impact factor: 0.522 (2016).

Alfonso Fernández, María Ana Saenz, Nestor Pérez. «Gestión metrológica en la Salud.» AENOR Revista de la normalización y la certifi cación, 2015: 38-43. ISSN: 2255- 0801.

A. Fernández, J.A. Robles Carbonell, P. Centeno de la Torre, G. Ruiz Córdoba, D. Arnal, R. Mur, D. Montes, M. Sáenz, P. Martínez Pallarés. La trazabilidad metrológica en la anestesia. e-medida - Revista Española de Metrología. vol. online, no. 10, Junio 2016 [Online].

Palancar-Martínez, D; Sáenz-Nuño, M. A. (Directora) Trabajo Fin de Master Propuesta de Norma Técnica para la Gestión metrológica en el Sector Sanitario Español, junio 2018. Univ. Pontifi cia Comillas de Madrid.

M. Sáenz, N. Pérez Mallada.’La biomecánica más avanzada se apoya en una metrología de calidad."e-medida - Revista Española de Metrología. vol. online, no. 5, Diciembre 2013 [Online].

UNE 66180:2008 “Sistemas de Gestión de calidad. Guía para la Gestión y la evaluación de los procesos de medición y de confi rmación metrológica”.

UNE EN ISO 10012:2003 “Sistemas de Gestión de las mediciones, requisitos para los procesos de medición y los equipos de medición". 